\title{
The Digital Lock-in Amplifier for Detecting the Power Traveling Wave Signal
}

\author{
Bo Ye*, Fei Chen and Ming Li \\ Engineering Research Center of Smart Grid, Yunnan Province, Faculty of Electric \\ Power Engineering, Kunming University of Science and Technology, Kunming \\ 650500, China \\ *corresponding author (yeripple@ @otmail.com)
}

\begin{abstract}
The traditional detection methods are easily affected by noise. It is difficult to extract the useful signal from the power traveling wave which is submerged in a lot of noise. A method using digital lock-in amplifier is proposed for detecting the power traveling wave signal. The basic principles of the lock-in amplifier are studied. Virtual instrument language LabVIEW is used to design the digital lock-in amplifier. It is able to extract the corresponding frequency components of the power traveling wave signal. As the front panel and the flow sheet of the digital lock-in amplifier are presented, the design of each module is introduced in detail. The experimental research on detecting signals containing noise and not containing noise are carried out, respectively. The results of experiment show that the presented digital lock-in amplifier can accurately extract the corresponding frequency components of the power traveling wave signal. Moreover, with the increase of the noise amplitude, the root mean square error of the output signals varies little. The presented digital lock-in amplifier is able to cancel the effect of noise. Therefore, it is a powerful tool to detect and extract the power traveling wave signal.
\end{abstract}

Keywords: traveling wave, signal detection, digital lock-in amplifier, LabVIEW, virtual instrument

\section{Introduction}

A large number of random noises exist in the work environment of the travelling-wave fault location device, restricted by transformer bandwidth and the analysis method. Therefore, it is difficult to separate the noise and useful signal. In process of collection and analysis, the traveling wave signals along with high frequency and weak amplitude are more susceptible to noise interference. It will cause the extracted traveling wave signal data not accurate and have impact on the position accuracy [1-6]. In Literature [7], we design a kind of filter installing in line roadside in order to obtain the wave signal, which combine capacitive voltage transformer and high-voltage coupling capacitor, but the filtering effect is not obvious, traveling wave signal is still affected by noise interference. In Literature [8], we adopt the method of narrowband filtering to restrain the high-frequency component from line outside, by adjusting the filter pass-band. We can make it just as the resistance filter to achieve filtering, but it can only get the traveling wave signal in the pass-band instead of getting the full wave signal. In Literature [9], In view of the traditional capacitive voltage transformer, we know that it can't pass and change the transient high-frequency signal, we can use capacitive voltage transformer or the casing end screen capacitance of the current transformer to extract voltage traveling wave. In Literature [10], it adopts the method of mathematical morphology to achieve the suppression of noise interference and extract traveling wave signal. The results were slightly better than the wavelet algorithm, but after de-noising, the wave-head steepness is 
reduced, the traveling wave signal amplitude is weakened. In Literature [11], by getting the cable's current traveling wave signal by the hall sensor, data acquisition card work in fault-current traveling wave to conduct high-speed sampling and storage control, but it still did not consider the errors caused by noise.

The lock-in Amplifier is a kind of device which act on alternating signal phase sensitive detection and use the filter device to restrain noise interference, it can effectively extract the useful signal from the signal containing noise, it has such advantages as high sensitivity, strong processing capacity, simple operation, precision advanced features, etc., [12, 13], but the lock-in amplifier's development cycle is long, is difficult to maintenance, versatility is not strong, greatly influenced by environmental factors in actual application. Virtual instrument language LabVIEW With features strong commonality, portable, easy maintenance, etc., [14] It is available to develop digital Lock-in Amplifier to replace the current chip-style Lock-in Amplifier, and it also further improves the precision of the Lock-in Amplifier and expands its generality.

Therefore, this paper proposes the application of the digital lock-in amplifier to power traveling wave signal detection, based on the fundamental principle of lock-in amplifier analysis and research. Under the help of virtual instrument language LabVIEW, we can design the digital lock-in amplifier whose detection signals corresponding to the frequency of the traveling wave signal. The digital lock-in amplifier block diagram and front panel have given. The block diagram gives a detail description about the design of each module. Finally, we carry out detecting experiment on the traveling wave signal containing noise and not containing noise. The detection results were analyzed and compared.

\section{The Basic Principle of Lock-in Amplifier}

The Lock-in amplifier is based on the cross-correlation signal detection. It is a kind of device which adopts Cross-correlation calculation between the detected signal and the same frequency reference signal to achieve signal detection $[15,16]$. Due to various signals component is included in the detected signal, the lock-in amplifier adopts heterodyne oscillation technology, transforming the signal component which same as the reference signal frequency into the DC signal, while the other frequency components are converted into AC signal, filtered by the low- pass filter. Accordingly, the lock-in amplifier applies phase-sensitive detection to detect the same frequency component between the signal containing noise and the reference signal. It can extract the frequency component corresponding to the traveling wave signal from a large number of noise signals. The principle of Lock-in amplifier is shown in Figure 1.

Supposing the input signal is $V_{i}(t)$, the reference signal is $V_{R 1}(t)$ and $V_{R 2}(t)$. The amplitude of the output signal is $V_{s 1}$. The output signal phase is $\theta$. PSD1 and PSD2 are multipliers. LPF is a low-pass filter. I and Q are low-pass filter output signal.

Supposing the input signal is:

$$
V_{i}(t)=V_{s 1} \sin \left(\omega_{1} t+\phi_{1}\right)
$$

Where t represents time, $\omega_{1}$ is the angular frequency of the signal $V_{i}(t), \phi_{1}$ is the initial phase angle of the signal $V_{i}(t)$. 


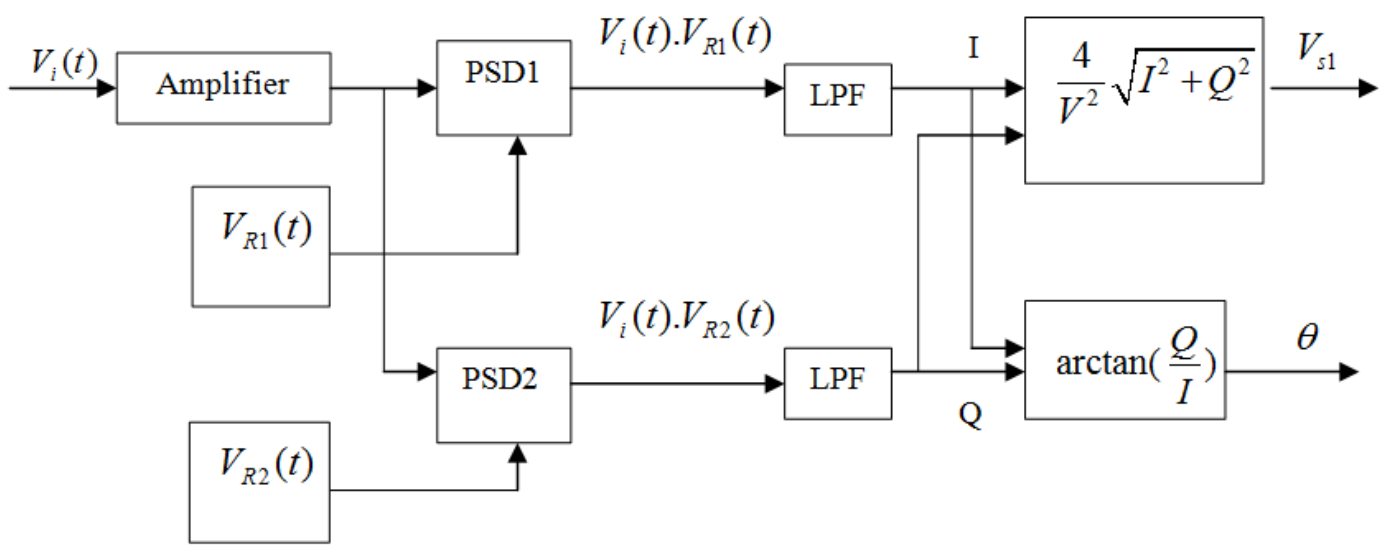

Figure 1. Block Diagram of Digital Lock-in Amplifier

Assuming the initial phase angle of the reference signal $V_{R 1}(t)$ and $V_{R 2}(t)$ is 0 ,

$$
\begin{aligned}
& V_{R_{1}}(t)=V_{1} \sin \left(w_{2} t\right) \\
& V_{R_{2}}(t)=V_{2} \cos \left(w_{2} t\right)
\end{aligned}
$$

Where $V_{1}$ is the amplitude of the signal $V_{R 1}(t), V_{2}$ is the amplitude of the signal $V_{R 2}(t)$, the signal $V_{R 1}(t)$ and $V_{R 2}(t)$ in the same frequency, angular frequency both are $\omega_{2}$.

Assuming $V_{1}=V_{2}=V, \omega_{1}=\omega_{2}=\omega$, multiplying the input signal with the reference signal, equivalent to the signal frequency migration, then

$$
\begin{aligned}
V_{i}(t) V_{R 1}(t) & =V_{s 1} V_{1} \sin \left(w t+\phi_{1}\right) \sin (w t) \\
& =V_{s 1} V / 2\left[\cos \phi_{1}-\cos \left(2 w t+\phi_{1}\right)\right] \\
V_{i}(t) V_{R 2}(t) & =V_{s 1} V_{2} \sin \left(w t+\phi_{1}\right) \cos (w t) \\
& =V_{s 1} V / 2\left[\sin \phi_{1}+\sin \left(2 w t+\phi_{1}\right)\right]
\end{aligned}
$$

By the use of low-pass filter, we can filter the AC component and retain the DC component. The low-pass filter output signal is :

$$
\begin{aligned}
& \mathrm{I}=V_{s 1} V / 2 \cos \phi_{1} \\
& \mathrm{Q}=V_{s 1} V / 2 \sin \phi_{1}
\end{aligned}
$$

The calculated amplitude and phase of the input signal:

$$
\begin{aligned}
& V_{s 1}=\frac{4}{V^{2}} \sqrt{I^{2}+Q^{2}} \\
& \theta=\arctan \left(\frac{I}{Q}\right)
\end{aligned}
$$

By the equations (4)-(9), when the lock-in amplifier's input signal and the reference signal are no phase difference, the lock-in amplifier can accurately measure the magnitude of the input signal. When the input phase signal and the reference signal have phase difference, the lock-in amplifier can be used to determine the phase difference between input signal and the reference signal. Thus, by appropriately adjusting the lock-in amplifier parameters, it can accurately obtain the amplitude and phase of the input signal, using of the amplitude and phase, we can restore the corresponding frequency of the signal.

\section{Digital Lock-in Amplifier Design}

LabVIEW is a graphical programming language widely used in the industrial, academic and various types of laboratory [17]. It owns a short development cycle, strong commonality, high degree of visual, intuitive operation, functional changes the 
advantages of simple, strong commonality, easy to maintain [14]. Provided with virtual elements, we can design the general digital lock-in amplifier used to detect the traveling wave signal of the corresponding frequency. Digital lock-in amplifier is composed of the front panel and program block diagram, the following paper respectively for the design details of the front panel and program block diagram.

\subsection{The Digital Lock-in Amplifier Front Panel}

Front panel located in the digital lock-in amplifier is divided into a signal generation panels, control panels and signal extraction panels of three-part sections. Signal generation panel is composed of the frequency knob, amplitude knobs, phase knobs, noise amplitude knob and reset button. Respectively control the input signal frequency, amplitude, phase, and noise amplitude, the reset button controls the program to start or stop. Control panel consists of a sliding lever knob, frequency knob and direct numerical output display section, the sliding lever control filter's the Low-pass cut-off frequency and frequency knob controls the size of the reference signal the frequency, numerical output section display reference signal phase and amplitude. Signal extraction panel is composed of signal-Vector measurement value output, single-frequency measured values output. The panel can display the input signal waveform and the output signal waveform. It also can export vector measurement of the related numerical, single frequency measurement output amplitude, frequency and phase. The designed front panel of digital Lock-in amplifier is shown in Figure 2.

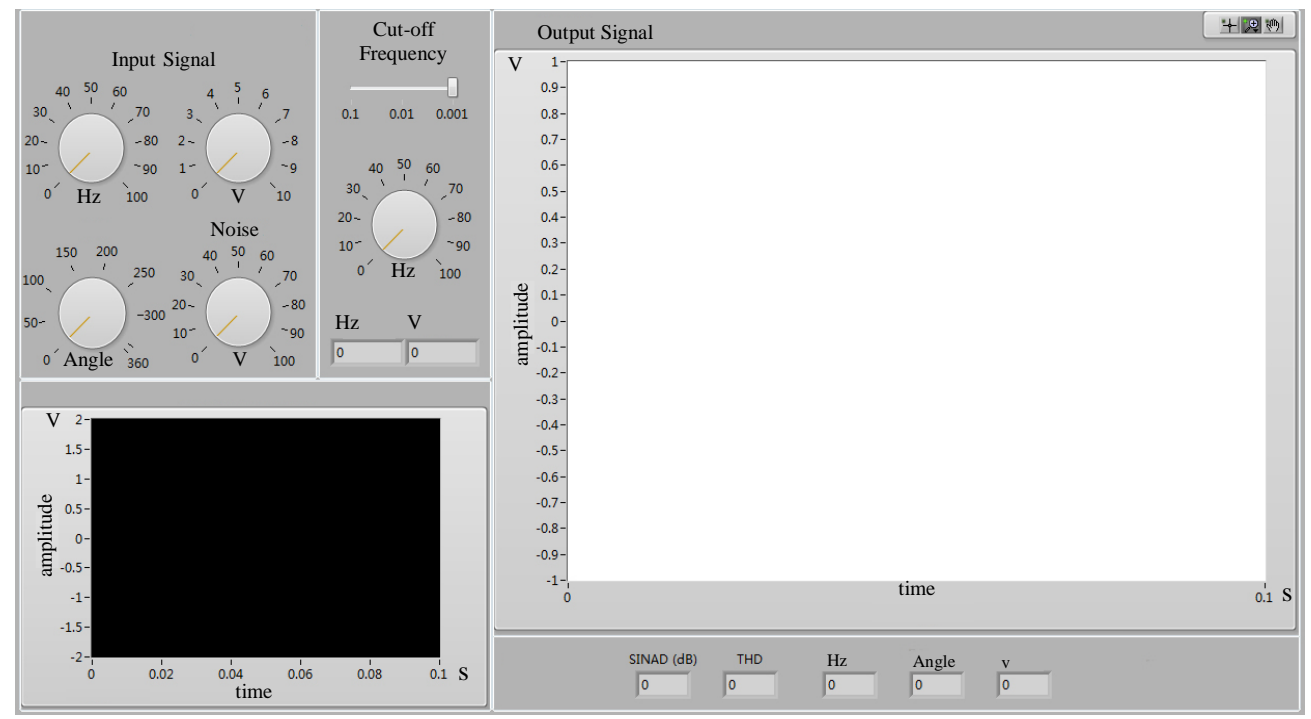

Figure 2. Front Panel of Digital Lock-in Amplifier

\subsection{Digital Lock-in Amplifier Block Diagram}

Digital lock-in amplifier block diagram shown in Figure 3, it is composed of the signal generating module, the pre-filtering module, the phase-sensitive detection module, the signal recovery module, the measured value display module of five parts. 


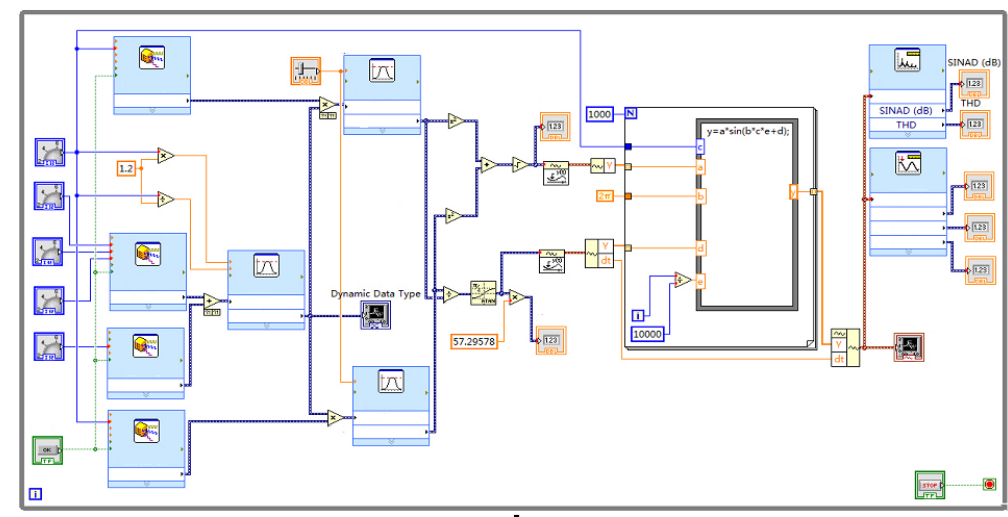

Figure 3. Flow Sheet of Digital Lock-in Amplifier

(1) The signal generation module

The input signals can be generated by "simulation signal" in Express palette. We can get the sinusoidal waveform of the given frequency, amplitude and phase by the help of simulation signal controls, and overlay several adjustable intensity on the waveform to simulate noise signal. As shown in Figure 4, Simulation signal 3 and 1, simulation signal 2 are the sine-wave signal generation modules, we can apply the signal frequency, phase and amplitude of three knobs to achieve parameters' adjustment. Simulation signal 4 is the uniform noise generating module. Its amplitude is adjusted by the noise amplitude knob of the front pane.

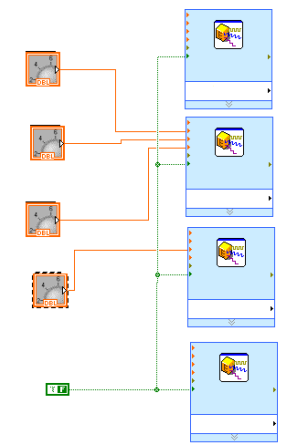

Figure 4. Signal Generation Module

(2) The Previous filtering module

When the digital lock-in amplifier is used to detect weak signals in strong noise or drowned in even greater noise, the previous filtering module preliminary filter the signal in the first place. A 9- step Bessel band-pass filter provided by LabVIEW is used in the filtering process, as shown in Figure 5. We can carry out linkage on the reference signal frequency and simulation signal, then the previous filtering module 3 conduct preliminary filtering, phase of the output signal and the input signal which both are from the previous filtering module is identical. The high cut-off frequency of band-pass filter is of 1.2 times the reference signal frequency. The low cut-off frequency is the reference signal frequency/1.2. 


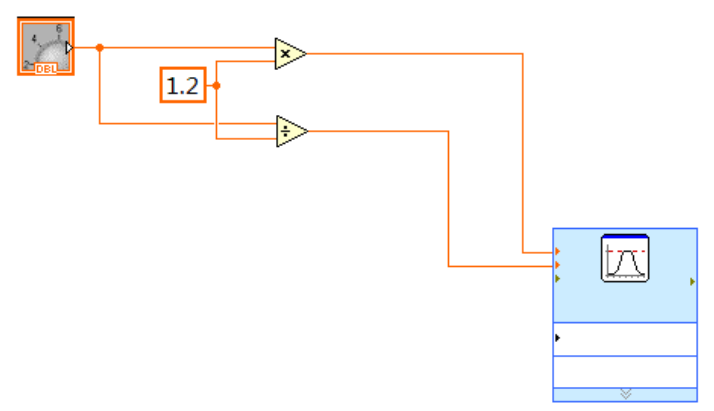

Figure 5. Previous Filtering Module

(3) The Phase-sensitive detection module

The phase-sensitive detection module consists of low-pass filter and Phase-sensitive detection section, as shown in Figure 6. The phase-sensitive detection module uses the low-pass filter with minimal bandwidth. The low-pass bandwidth can be adjusted, when the low-pass bandwidth is small, it possesses those features as high precision, strong noise suppression capability, but the response time is longer. When the low-pass bandwidth is large, it leads to low precision, lack in noise suppression capability, but the response time is shorter. Low-pass filter adopts the adjustable design. It is enough to adapt to input signal in the different signal-noise ratio. The Phase-sensitive detection section takes the relevant operation module according to the basic principle of the lock-in amplifier to the input signal and reference signal, in order to comply the corresponding operation. By this method, we can get the detected signal amplitude and phase information.

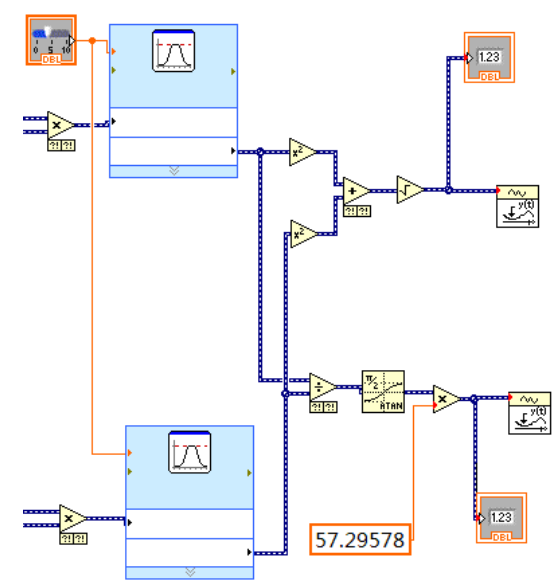

Figure 6. Phase-sensitive Detection Module

(4) The Signal recovery module

Based on the measured signal amplitude and phase, the signal recovery module may restore the corresponding sine signal, In order to carry out further analysis and identification on the traveling wave signal with the detected frequency, as shown in Figure 7. First, the signal is converted to a double-precision array format from dynamic mode, then calculate reduction signal using the formula point-by-point. 


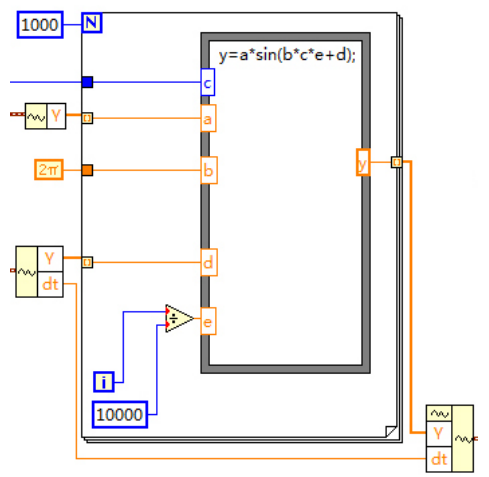

Figure 7. Signal Recovery Module

(5) The Measurement display module

The processed and calculated data display in the Measurement display module, as shown in Figure 8. The display includes:

Amplitude and phase: Where $\varphi$ is the phase difference between the measured signal and reference signal, $\mathrm{U}$ is the measured signal amplitude. As a result of adopting the orthogonal vector lock- in amplifier, instead of adjusting the frequency of the reference signal, it can obtain amplitude and phase.

Signal waveform: display waveform of the reduction signal and analyze the reduction effect, list signal-to-noise ratio of the signal, harmonic distortion and other indicators, evaluate performance of the phase-locked amplifier.

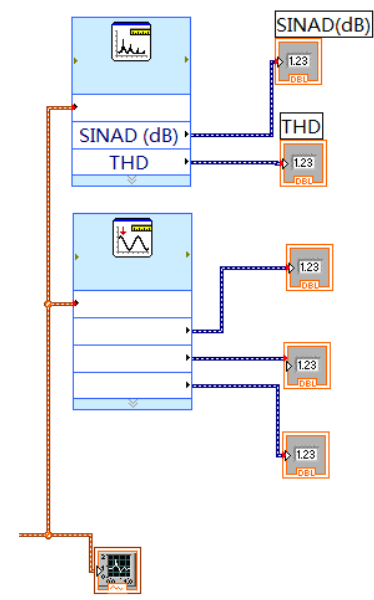

Figure 8. Measurement Display Module

\section{Experimental Results and Analysis}

\subsection{Sine Signal Detection}

In order to test the designed digital lock-in amplifier to extract the useful signal from the signal containing a lot of noise, we can take advantage of sinusoidal signal to test, the sinusoidal signal frequency is $100 \mathrm{~Hz}$. Amplitude is $1 \mathrm{v}$. The phase angle is 0 , submerged in the amplitude of $100 \mathrm{v}$ white gaussian noise. Adjusting the front panel knob at the moment, the frequency knob to $100 \mathrm{~Hz}$, the amplitude knob to $1 \mathrm{v}$, the phase knob to $0^{\circ}$, noise amplitude knob to $100 \mathrm{v}$, getting sinusoidal signal drowned in the noise as shown in Figure 9. 


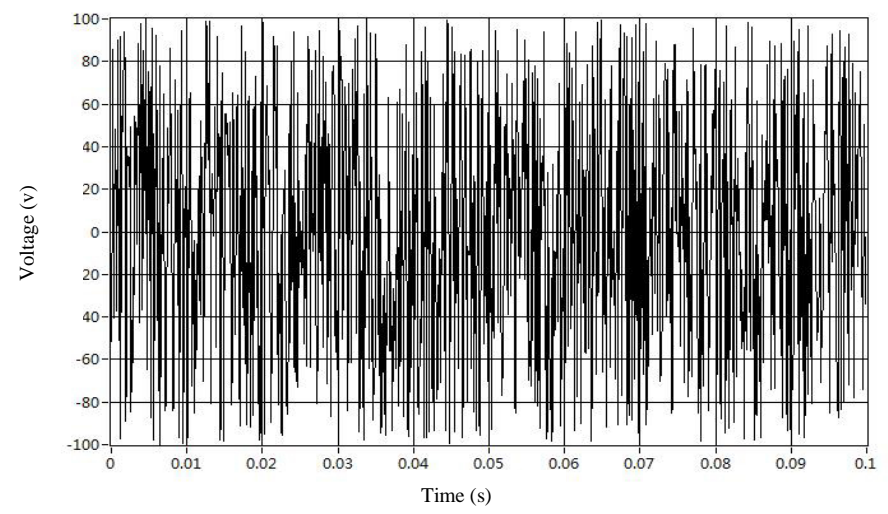

Figure 9. Sinusoidal Wave Covering by Noise

The cut-off frequency of low-pass filter in the regulation program diagram is set to $0.001 \mathrm{~Hz}$. The output waveform is shown in Figure 10.

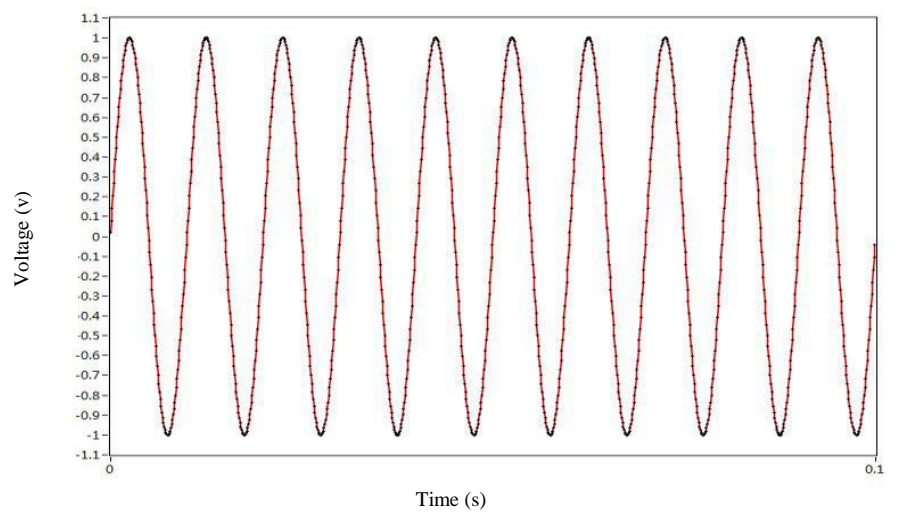

Figure 10. Output Waveform

Watching numerical results on the front panel display, amplitude of the output waveform is $1.00187 \mathrm{v}$. Frequency is $100 \mathrm{~Hz}$. Phase angle is $1.0419^{\circ}$. Signal noise ratio is $113.306 \mathrm{~dB}$. Total harmonic distortion is $1.2839 \times 10^{-9}$.

By comparing the input waveform with the output waveforms, we can draw a conclusion that the digital lock-in amplifier can be designed to extract accurate sinusoidal signal buried in the noise.

\subsection{Traveling Wave Signal Detection}

(1) The traveling wave signal detection excluding noise

Setting the traveling wave hits for 3000, acquisition for traveling wave signal excluding noise as shown in Figure 11. 


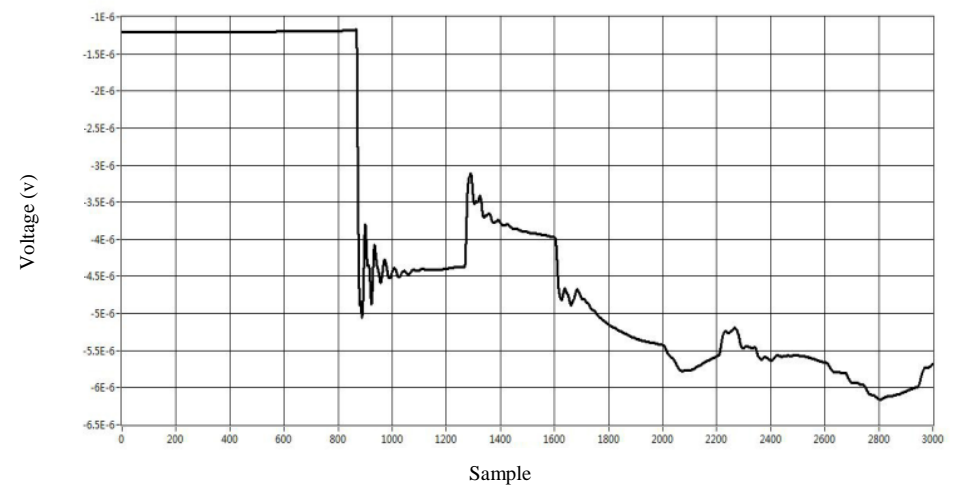

Figure 11. Traveling Wave Signal not Containing Noise

Then set reference frequency knob of control panel in the digital lock-in amplifier to $4.33 \mathrm{kHz}$, the cut-off frequency of Low-pass filter is set to $3 \mathrm{kHz}$, cancel a band-pass Prefilter and obtain the output waveform shown in Figure 12, root mean square error of the output signal is $3.11199 \times 10^{-6}$.

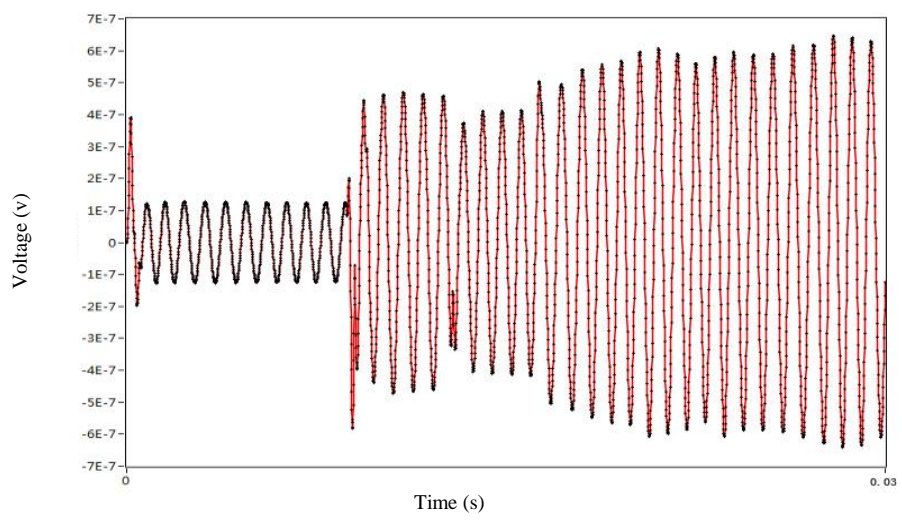

\section{Figure 12. Sinusoidal Component Extracting from the Travelling Wave Signal not Containing Noise}

(2) Traveling-wave signal detection containing noise

Based on the above experiment, adding the noise signal amplitude of $10^{-6} \mathrm{v}$ to the traveling wave, the traveling wave signal containing noise is shown in Figure 13. Sine component extracted from the traveling-wave signal containing noisy shown in Figure 14, root mean square error of the output signal is $3.12655 \times 10^{-6}$.

To further increase the noise amplitude to $2 \times 10^{-6} \mathrm{v}$, the traveling wave signal containing noise as shown in Figure 15, the extracted sine component shown in Figure 16, the root mean square error of the output signal is $3.27447 \times 10^{-6}$.

(3) The experiment results analysis

When the input signal is traveling wave signal not containing noise, the designed lockin amplifier can accurately extract sine component of corresponding frequency from traveling Wave signal, the root mean square error of the output signal is $3.11199 \times 10^{-6}$. After adding the noise signal amplitude of $10^{-6} \mathrm{v}$ to the input signal, output waveform of lock-in amplifier get without obvious changes, the root mean square error of the output signal is $3.12655 \times 10^{-6}$. But the noise amplitude is double sized as the original real signals, the root mean square error of the output signal is $3.27447 \times 10^{-6}$. From here we see that along with the increase of noise amplitude, the root mean square error of the output signal 
varies little, the designed digital lock-in amplifier has a strong anti-noise capability, it can accurately detect the traveling wave signal submerged by noise.

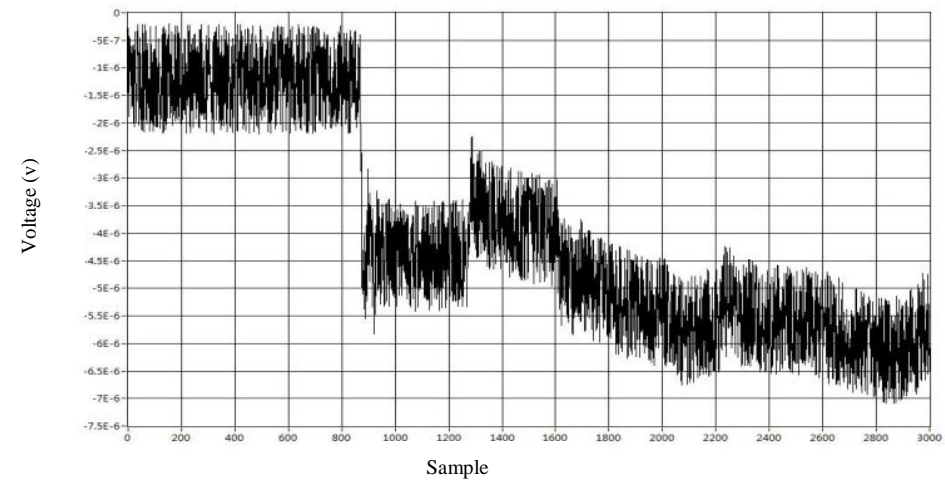

Figure 13. Traveling Wave Signal Containing Noise (Amplitude: $10^{-6} \mathrm{v}$ )

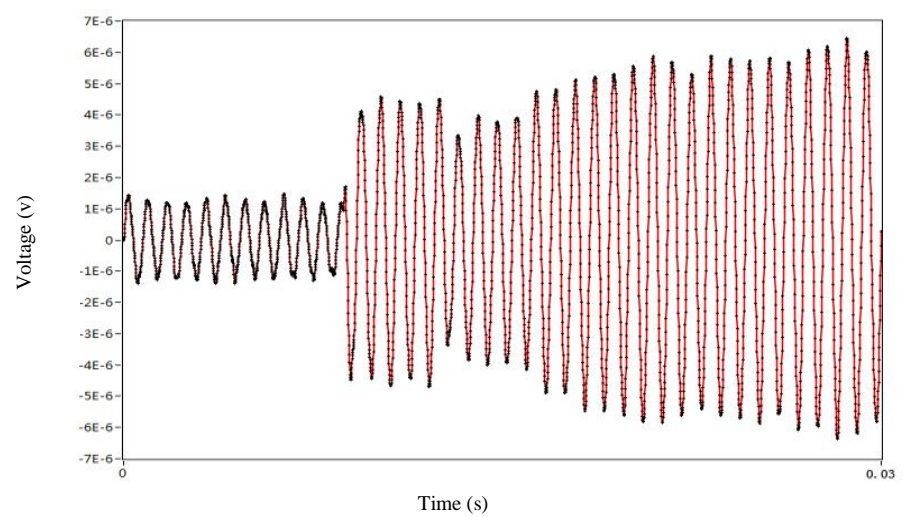

Figure 14. Sinusoidal Component Extracting from the Traveling Wave Signal Containing Noise (Amplitude: $10^{-6} \mathrm{v}$ )

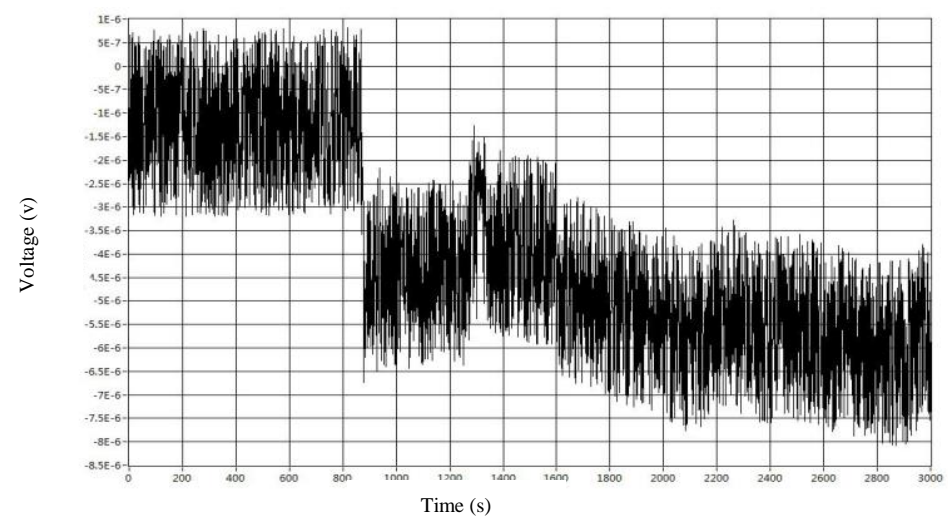

Figure 15. Traveling Wave Signal Containing Noise (Amplitude: $2 \times 10^{-6} \mathrm{v}$ ) 


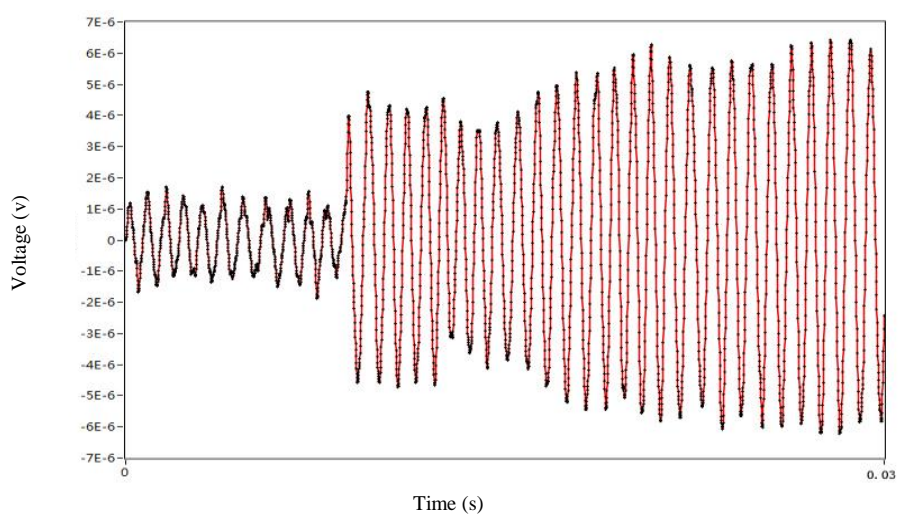

\section{Figure 16. Sinusoidal Component Extracting from the Traveling Wave Signal Containing Noise (Amplitude: $2 \times 10^{-6} \mathrm{v}$ )}

\section{Conclusion}

This paper studied the basic principle of the lock-in amplifier, applying Virtual Instrument language LabVIEW to design the digital lock-in amplifier which detects the traveling wave signal. Describing the functions about the corresponding various parts of the front panel and block diagram, this paper gives a detailed description of composition, role and setting for many modules of the program block diagram, such as the signal generating module, the Pre-filtering module, the Phase-sensitive detection module, the Signal recovery module, the measured value display module. By using the designed digital lock-in amplifier to test two kinds of traveling wave signal, ones is containing noise, another is not containing noise. As the experimental results shown, the designed digital lock-in amplifier can accurately extract the sinusoidal component of corresponding frequency in traveling wave signal, and with the increase of noise amplitude, root mean square error of the output signal varies little, it indicates that the designed digital lock-in amplifier has a strong anti-noise capability.

In practical application, because the travelling wave is the high-frequency signal, it is difficult to determine wave frequency range, after the checksum, the designed digital lock-in amplifier is better to extract $5 \mathrm{kHz}$ sinusoidal component. We further intend to make an improvement on the design of the system parameters, making it conform to the requirements of the traveling wave signal extraction in various frequencies.

\section{ACKNOWLEDGEMENTS}

This work is supported by the National Natural Science Foundation of China Grant No. 51465024, 51105183, the Research Fund for the Doctoral Program of Higher Education of China Grant No. 20115314120003, the Applied Basic Research Programs of Science and Technology Commission Foundation of Yunnan Province of China Grant No. 2010ZC050, the Foundation of Yunnan Educational Committee Grant No. 2013Z121, the Science and Technology Project of Yunnan Power Grid Corporation Grant No. K-YN2013-110.

\section{References}

[1] L. Wu, Z. He and Q. Qian, "Single ended fault location method using traveling wave natural frequency", Proceedings of the Chinese Society of Electrical Engineering, vol. 28, no. 10, (2008), pp. 69-75.

[2] C. Christopoulos, D. W. P. Thomas and A. Wright, "Signal processing and discriminating techniques incorporated in a protective scheme based on travelling waves", IEE Proceedings C: Generation Transmission and Distribution, vol. 136, no. 5, (1989), pp. 279-288.

[3] V. Siozinys and R. Urniezius, "Transmission line protection and fault location based on travelling wave measurement”, Elektronika Ir Elektrotechnika, vol. 19, no. 9, (2013), pp. 21-24. 
[4] S. Darren and J. Zhu, "Improved single ended traveling wave fault location algorithm based on experience with conventional substation transducers", IEEE Transactions on Power Delivery, vol. 21 no. 3, (2006), pp. 1714-1720.

[5] I. Ashraf, A. Megahed, M. Moussa and A. E. Bayoumy, "Usage of wavelet transform in the protection of series compensated transmission lines," IEEE Transactions on Power Delivery, vol. 21 no. 3, (2006), pp. 1213-1221.

[6] A. Abur and F. Magnago, "Fault location using wavelets," IEEE Transactions on Power Delivery, vol. 13, no. 4, (1988), pp. 1475-1480.

[7] A. T. Johns and P. Agrawal, "New approach to power line protection based upon the detection of fault induced high frequency signals", IEE Proceedings C: Generation Transmission and Distribution, vol. 137, no. 4, (1990), pp. 307-313.

[8] X. Jiang and A. M. Gole, "A frequency scanning method for the identification of harmonic instability in HVDC systems", IEEE Transaction on Power Delivery, vol. 10, no. 4, (1995), pp. 1875-1881.

[9] G. Zou, H. Gao, M. Xu and B. Xu, "Method for extracting transient voltage travelling wave signal from high voltage power grid", Automation of Electric Power Systems, vol. 33, no. 2, (2009), pp. 71-74.

[10] J. Wu, Y. Wu, J. He and W. Yang, "Application of mathematical morphology in transmission line fault location", Relay, vol. 33, no. 17, (2005), pp. 21-26.

[11] J. Duan, "Cable fault traveling wave on line monitoring system based on LabVIEW", Instrument Technique and Sensor, vol. 12, (2011), pp 43-45.

[12] J. Gaspar, S. Chen, A. Gordillo, M. Hepp, P. Ferreyra and C. Marques, "Digital lock in amplifier: Study, design and development with a digital signal processor", Microprocessors and Microsystems, vol. 28, no. 4, (2004), pp. 157-162.

[13] M. O. Sonnaillon, R. Urteaga and F. J. Bonetto, "High-frequency digital lock-in amplifier using random sampling", IEEE Transactions on Instrumentation and Measurement, vol. 57, no. 3, (2008), pp. 616-621.

[14] C. J. Kalkman, "LabVIEW: A software system for data acquisition, data analysis, and instrument control”, Journal of Clinical Monitoring, vol. 11, no. 1, (1995), pp. 51-58.

[15] S. A. Jacobson, P. N. Denbigh and D. E. H. Naude, "A new method for the demodulation of ultrasonic signals for cross-correlation flowmeters", Ultrasonics, vol. 23, no. 3, (1985), pp. 128-132.

[16] G. V. Duong, R. Groessingera, M. Schoenhart and D. Bueno-Basquesc, "The lock-in technique for studying magnetoelectric effect”, Journal of Magnetism and Magnetic Materials, vol. 316, no. 2, (2007), pp. 390-393.

[17] R. H. Bishop, Editor, “Learning with LabVIEW 2009”, Prentice Hall, (2009).

\section{Copyright forms}

You must include your fully-completed, signed SERSC copyright release form when you submit your paper. WE MUST HAVE THIS FORM BEFORE YOUR PAPER CAN BE PUBLISHED IN THE PROCEEDINGS. The copyright form is available from journal home page. Authors should send their copyright forms to FAX. +82-42-624-2205 or Email sersc@sersc.org

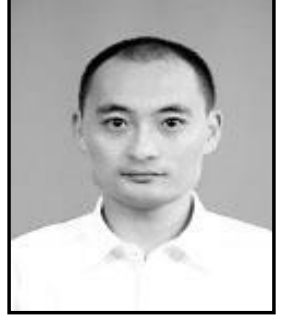

Bo Ye, He is an associate professor in Faculty of Electric Power Engineering, Kunming University of Science and Technology. He received the B.E. degree in electrical engineering from Kunming University of Science and Technology in 2000 and received the Ph.D. degree in control science and engineering from Zhejiang University in 2009. His research interests are statistical learning, artificial intelligence, computational electromagnetic modeling, and power system protection and control.

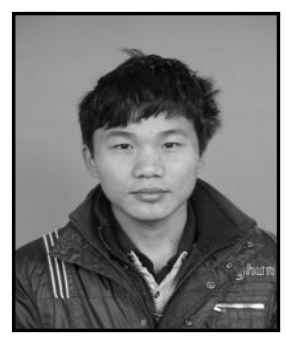

Fei Chen, He is a master candidate in Faculty of Electric Power Engineering, Kunming University of Science and Technology. He received his B.E. degree in electrical engineering from Kunming University in 2013. His research interests are Power system fault analysis and Eddy Current testing. 


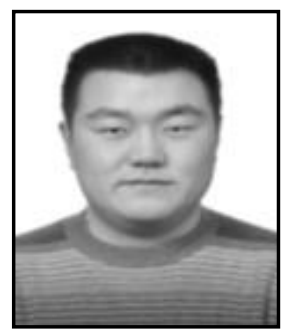

Ming Li, He is a master candidate in Faculty of Electric Power Engineering, Kunming University of Science and Technology. He received the B.E. degree in electrical engineering from Nanjing University of Science and Technology in 2010. His research interests are electrical testing technology and development of non-destructive testing equipment. 
International Journal of Signal Processing, Image Processing and Pattern Recognition Vol. 8, No. 4 (2015) 